\title{
Dissociation of indirect excitons: Discontinuity and bistability in the tunnel current of single-barrier heterostructures
}

\author{
A. Parlangeli, P. C. M. Christianen, A. K. Geim, and J. C. Maan \\ Research Institute for Materials, High Field Magnet Laboratory, University of Nijmegen, Toernooiveld 1, \\ 6525 ED Nijmegen, The Netherlands \\ L. Eaves, P. C. Main, and M. Henini \\ Department of Physics, University of Nottingham, NG7 2RD Nottingham, United Kingdom
}

(Received 6 July 1999)

\begin{abstract}
We observed a new discontinuity and bistability in the tunnel current of a 12-nm single-barrier GaAs/AlAs $p-i-n$ heterostructure where a system of spatially separated two-dimensional electron and hole $(e-h)$ layers of equal and tunable density is realized. Both features appear at $T \$ 300 \mathrm{mK}$ and are substantially enhanced in a magnetic field $B \gtrsim 10 \mathrm{~T}$ perpendicular to the layers. They correspond to a discontinuity in the $e-h$ density and in the phase of the current magneto-oscillations, which we suggest to arise from a transition between indirect excitons and the uncoupled $e-h$ gases. [S0163-1829(99)12343-9]
\end{abstract}

The Coulomb coupling between spatially separated twodimensional (2D) electron-hole $(e-h)$ layers of equal density has been a topic of intense study in the last few years. ${ }^{1-9}$ In these systems, the overlap between the electron and hole wave functions can be made arbitrarily small, and thus the recombination lifetime of the carriers can be drastically increased with respect to the strictly $2 \mathrm{D} e-h$ system. Thermalization of nonequilibrium carriers to the lattice temperature is thus achievable, and-depending on interlayer distance, density, applied magnetic field and temperature-the electronic configuration is theoretically expected to range from the normal exciton gas to the excitonic state 2,3 or the separate fractional quantum Hall states. ${ }^{4,5}$ Despite the marked interest, it is only recently that technology has made available semiconductor heterostructures where closely spaced 2D $e$ and $h$ layers can be realized.

The most studied systems are double quantum wells $\mathrm{s}^{6,7}$ and $p-i-n$ single-barrier heterostructures. ${ }^{2,8,9}$ In the latter devices, under forward bias, electrons and holes accumulate at opposite sides of the barrier to form two 2D layers of equal density. The main advantages are the presence of only two interfaces (inferring high in-plane mobility), the possibility of tuning the density with the applied voltage, and the fact that for sufficiently thick barriers $(\sim 10 \mathrm{~nm}$ for GaAs/AlAs structures) recombination and tunneling times are much longer than the carrier relaxation time. These systems are therefore expected to reach thermodynamic quasiequilibrium, in which the carrier temperature is equal to the lattice one and the chemical potentials of the $e$ and $h$ gases are separately aligned to the ones in the respective doped regions. In such devices, Neves et al. ${ }^{8}$ have observed resonant tunneling via indirect exciton states, which manifests itself as sharp peaks in the low-temperature ( $T$ $\lesssim 10 \mathrm{~K}) \quad I(V)$ curves.

We have measured the tunnel current of similar samples in a regime of higher carrier densities, and we have observed discontinuity and bistability, which develop when the inplane interparticle distance $d_{e-e}$ becomes comparable to the interlayer separation $d_{e-h} \approx 25 \mathrm{~nm}$ (comparable the GaAs Bohr radius of $12 \mathrm{~nm}$ ).
The device under study is a 12 nm-thick single-barrier $p-i-n$ GaAs/AlAs/GaAs heterostructure, ${ }^{8,9}$ grown by molecular beam epitaxy in the [311] crystallographic direction in order to ensure high in-plane hole mobility. A schematic band diagram under forward bias is shown in the inset of Fig. 1. In this structure, the applied voltage is proportional to the chemical potential of the $e-h$ system $\mu_{e-h}=e V$, which is defined as the energy required to add an $e-h$ pair. In the range of biases of our interest, only the lowest heavy-hole and electron subbands are populated. The measured current is determined by electrons, since holes have a much smaller tunneling probability because of their bigger effective mass.

When $d_{e-e} \sim d_{e-h} \approx 25 \mathrm{~nm}$, we observe a bistability in the $I(V)$ characteristic at $T=70 \mathrm{mK}$ and $B=0$ (Fig. 1),

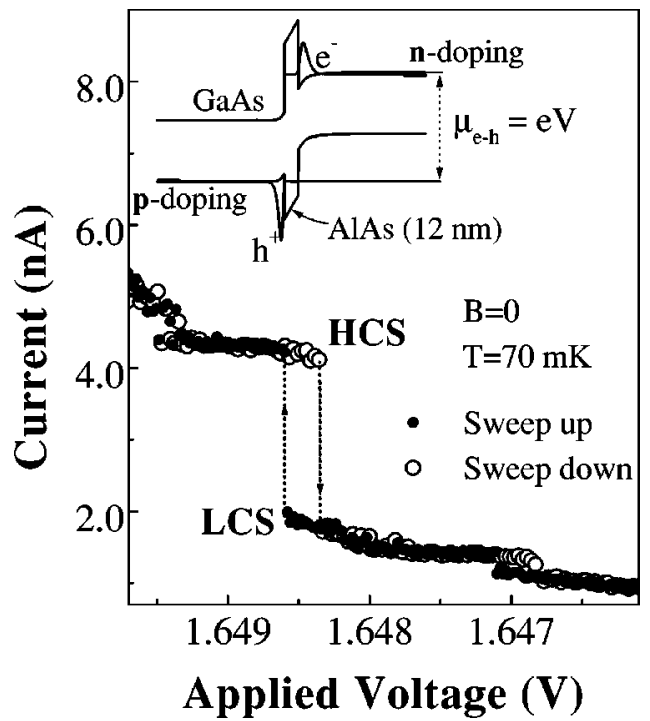

FIG. 1. Bistability of the tunnel current at $B=0$ and $T$ $=70 \mathrm{mK}$. The measured points are shown as filled and open circles for sweep up and down, respectively. The electronic states are named "high-current state" (HCS) in the upper branch and "lowcurrent state" (LCS) in the lower one. The inset shows a schematic band profile under forward bias. 

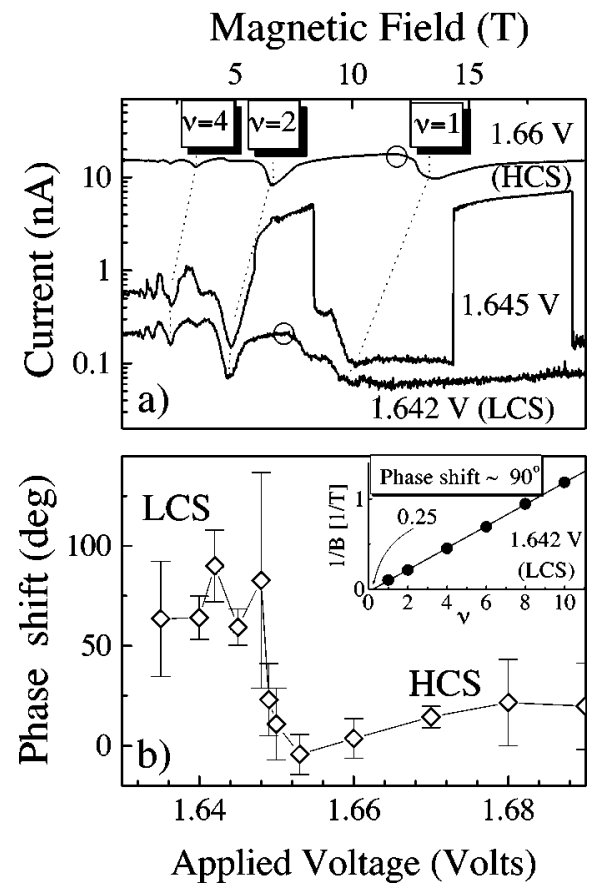

FIG. 2. (a) Magneto-oscillations (MO) traces for $V=1.66 \mathrm{~V}$ ("high-current state" or HCS), $V=1.642 \mathrm{~V}$ ("low-current state" or LCS) and $V=1.645 \mathrm{~V}$ (both LCS and HCS). Maxima positions at $1<\nu<2$ are marked by open circles. (b) Phase shift of the MO at fixed bias. The inset shows a plot of $1 / B$ vs $\nu$ for $V=1.642 \mathrm{~V}$.

which is accompanied by a discontinuity in the tunnel current of a factor of two. Both the bistability and the discontinuity disappear at temperatures higher than $T^{*} \sim 300 \mathrm{mK}$ $(\sim 30 \mu \mathrm{eV})$, but a smeared feature in the $I(V)$ survives up to $T=4.2 \mathrm{~K}$. Such behavior has not been observed on $n-i-n$ devices of similar structure (see, f.i. Refs. 10 and 11), and we believe that it originates from the presence of the additional hole layer. We denote the states corresponding to the upper and lower branch of the bistability "high-current state" (HCS) and "low-current state" (LCS), respectively (Fig. 1).

We have further characterized these states by measuring the current as a perpendicular magnetic field is swept at fixed applied voltage (i.e., at fixed chemical potential $\left.\mu_{e-h}=e V\right) .{ }^{10}$ In Fig. 2(a), three typical traces measured at $T=1.2 \mathrm{~K}$ are shown: one in the LCS $(1.642 \mathrm{~V})$, another in the HCS $(1.66 \mathrm{~V})$, and an intermediate one with both behaviors $(1.645 \mathrm{~V})$. These sweeps show general properties and do not strictly refer to Fig. 1, since the details of the bistability (position, width and current discontinuity) depend on temperature and magnetic field. The magneto-oscillations (MO) of the tunnel current arise from the effect of the magnetic field $B$ on the density of states of the two-dimensional electron gas (2DEG), which becomes quantized in Landau levels (LL's), with degeneracy $e B / h$, separated by the cyclotron energy $\hbar \omega_{c}=\hbar e B / m$ ( $m$ is the effective mass). The physical properties of the system are determined by the filling factor $\nu=n h / e B$ ( $n$ is the density), which is the number of occupied LL's. The Fermi energy $E_{F}$ of the 2DEG is pinned to both the last occupied LL and to the chemical potential (fixed by the applied voltage) in the doped regions. This condition induces a self-consistent rearrangement of the band profile when $B$ is swept ${ }^{10}$ which modulates the tunneling current with periodicity $E_{F} / \hbar \omega_{c} \propto 1 / B$. The minima occur

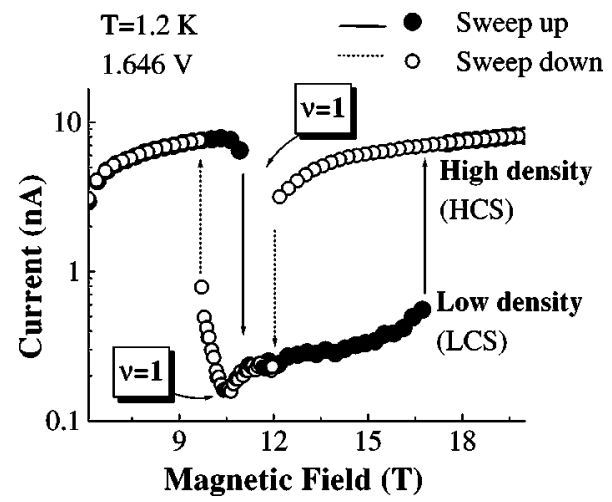

FIG. 3. Bistability in the tunnel current around $\nu=1$ under sweeping magnetic field up (filled circles) and down (open circles) at $V=1.646 \mathrm{~V}$ and $T=1.2 \mathrm{~K}$.

approximately at integer filling factor (we resolve spin splitting), at fields given by

$$
B_{\nu}=\frac{n h}{e \nu}=\frac{B_{1}}{\nu} .
$$

From Fig. 2(a) it is clear that the oscillations of LCS and HCS are quite different in nature, since the positions of the maxima differ considerably in the two states, as can be seen clearly for the maxima at $1<\nu<2$ [marked by circles in Fig. 2(a)]. We attribute this change in position to a change of the DOS, which is confirmed by the change of the periodicity of the MO. Indeed, the HCS oscillations can be described by Eq. (1), with a linear dependence of $n$ on $V$, as expected from a normal $2 \mathrm{DEG} .{ }^{12}$ On the contrary, the LCS shows a systematic deviation, and a linear $1 / B$ dependence can only be obtained when $\nu$ is replaced by $\nu-\alpha[0.2 \leq \alpha \leq 0.25$. See the inset of Fig. 2(b) for $V=1.642 \mathrm{~V}]$, corresponding to a phase shift of $60^{\circ}$ to $90^{\circ}\left(\alpha * 360^{\circ}\right)$ [Fig. 2(b) ]. Furthermore, in the bistable region, the LCS and the HCS show different densities at the same applied voltage (i.e., at the same chemical potential), as can be seen by sweeping $B$ around the $\nu=1$ minimum in both directions. In Fig. 3, we plot both curves together for $V=1.646 \mathrm{~V}$. We observe that the minimum in the MO is realized at a lower field (thus lower density) for the LCS $(10.5 \pm 0.1 \mathrm{~T})$ than for the HCS $(11.5 \pm 0.5 \mathrm{~T})$, and that the difference between the densities is about $10 \%$.

In order to gain further insight into the nature of the bistability, we measured how the hysteresis in the $I(V)$ curves at $B=0$ (Fig. 1) evolves under a constant magnetic field perpendicular to the layers (Fig. 4). The most striking property is an increase in both amplitude and width of one order of magnitude for $B \gtrsim 6 \mathrm{~T}$ [Fig. 4(a) for $B=10 \mathrm{~T}$ and $T$ $=70 \mathrm{mK}$ ], whereas a marked suppression occurs around the integer filling factors of the HCS, as shown in Fig. 4(a) (B $=11.5 \mathrm{~T}$ orresponds to $\nu \approx 1$ ). The width of the bistability [Fig. 4(b)], its voltage position, and the size of the discontinuity oscillate with $1 / \mathrm{B}$ periodicity.

We have also studied the stability and the temperature dependence at $B=10 \mathrm{~T}$. Both the LCS and the HCS are stable under a constant bias; but if we apply a fixed current in the region of negative differential conductance (1 to 5 nA), we observe that $V$ is unstable between the two values $(1.646$ and $1.653 \mathrm{~V})$ at which the discontinuity occurs [Fig. 

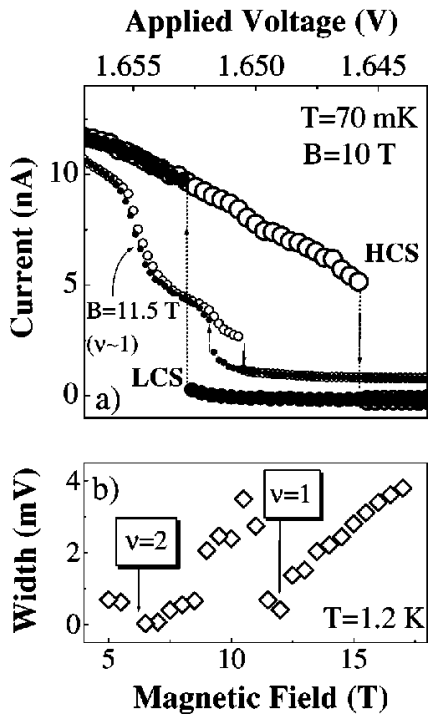

FIG. 4. (a) Bistability of the tunnel current at $B=10$ and $T$ $=70 \mathrm{mK}$. The filled and open circles refer to sweep up and down, respectively. The smaller circles (offset for comparison) refer to $B$ $=11.5 \mathrm{~T}(\nu \approx 1)$. (b) Width of the bistability as a function of magnetic field.

4(a)]. Under an increase in temperature, the bistability reduces in size and shifts to lower biases and higher currents. We observe the bistability up to $T=4.2 \mathrm{~K}$, which points to an energy scale of the order of $1 \mathrm{meV}$.

Finally, we have measured the influence of an in-plane magnetic field $B_{\|}$, at $T=1.2 \mathrm{~K}$. In this geometry, the HCS current decreases when $B_{\|}$increases, whereas the LCS current increases for $0.25 \lesssim B_{\|} \lesssim 1 \mathrm{~T}$, and decreases $B_{\|} \gtrsim 1 \mathrm{~T}$. The HCS behaves as a $2 \mathrm{DEG},{ }^{13}$ whereas the current increase of the LCS is anomalous. As a result of this difference between LCS and HCS, the feature in the $I(V)$ curves at $B$ $=0$ is clearly smeared by in-plane fields $B \geq 0.25 \mathrm{~T}$.

As we discuss below, all these experimental observations cannot be explained by previously reported mechanisms of bistability. Impurity-related trapping-detrapping processes are known to lead to bistability in bulk GaAs; ${ }^{14}$ in our case, however, the observed dependence on filling factor and the suppression under a modest in-plane magnetic field unambiguously show a 2D origin. Nor can the bistability be ascribed to trapping-detrapping mechanisms involving 2D states, as could arise from resonant tunneling via $X$-valley quantum well states or impurity states in the AlAs layer. We do observe the former as much weaker and broader features in the $I(V)$ at higher bias $(V \approx 1.7 \mathrm{~V}$, in agreement with Ref. 15). Impurity states in the barrier have been studied in $12 \mathrm{~nm}$ GaAs/AlAs/GaAs $n-i-n$ single-barrier structures with intentional Si doping in the AlAs layer; ${ }^{11}$ the $I(V)$ curves of these samples show broad tunneling features that evolve into peaks under a perpendicular magnetic field and shift to lower voltages for $B \gtrsim 10 \mathrm{~T}$, whereas the feature that we observe is bistable, discontinuous and does not shift globally in voltage under magnetic fields up to $17 \mathrm{~T}$.

It is more natural to explain our data in terms of the Coulomb attraction between the $e$ and $h$ 2D layers. Indeed, at low densities $\left(d_{e-e} \gg d_{e-h}\right)$, the system is a diluted gas of spatially indirect excitons, ${ }^{8}$ whereas at high densities $\left(d_{e-e}\right.$ $\lesssim d_{e-h}$ ) the $e$ and $h$ layers uncouple into separate 2DEG and 2DHG. ${ }^{12}$ We identify the HCS with the uncoupled $e$ and $h$ gases, since they behave regularly under perpendicular ${ }^{12}$ and in-plane ${ }^{13}$ magnetic fields. In contrast, the LCS behave peculiarly, and we believe that they can be described as indirect excitons.

This assignment is supported by the phase shift of the MO, since a similar behavior has been observed by Volkov et $a{ }^{16}{ }^{16}$ in the optical spectra of direct excitons and explained by the presence of exciton states below the free-particle LL's. ${ }^{17}$ We stress that our experimental results are remarkably consistent with a Fermi-Dirac distribution of the indirect excitons, in agreement also with Ref. 6. This behavior, which is nontrivial since isolated excitons are Bose particles, arises from the short-range dipole-dipole repulsion, which does not allow excitons to be in the same spatial position.

With the same simple model, we can explain the low current of the LCS at $B=0$, since the exciton states have lower density (Fig. 3) and smaller tunneling probability than the HCS. Indeed, the tunneling probability is proportional to the in-plane overlap between the wave functions of initial (LCS or HCS) and final states (plane-wave electrons in the collector). ${ }^{18}$ The overlap is bigger for the plane-wave HCS than for the LCS, because tunneling electrons in the LCS are confined in-plane by Coulomb interaction within an area of radius $\sim a_{B} \cdot{ }^{1,18}$. The LCS current increase under moderate magnetic field $B_{\|}$does not violate the preceding interpretation, but we are not able to quantify the effect of $B_{\|}$on the tunneling of indirect excitons.

In our interpretation, the LCS is characterized by a binding energy $E_{b}$, which we estimate from the temperature $T^{*}$ that suppresses both the bistability and the discontinuity. For $B=0$ and $B=10 \mathrm{~T}, T^{*}$ was shown to be $\sim 300 \mathrm{mK}$ and $\sim 10 \mathrm{~K}$, respectively, and roughly proportional to the bistability width (which saturates for $T \$ 300 \mathrm{mK}$ ). We can therefore estimate the ratio $E_{b}(10 \mathrm{~T}) / E_{b}\left(\begin{array}{ll}0 \mathrm{~T}\end{array}\right) \sim 20$. It is more difficult to estimate the absolute value of $E_{b}$ since the proportionality factors are not known a priori. With a leverage factor of 1 , we obtain a reasonable estimation of an upper limit: $E_{b} \sim 0.3 \mathrm{meV}$ at $B=0$ and $B \sim 5 \mathrm{meV}$ at $B$ $\sim 10 \mathrm{~T}$; if we assume that $E_{b} \sim T^{*}$, we find a lower limit: $E_{b} \sim 0.03 \mathrm{meV}$ at $B=0$ and $B \sim 0.5 \mathrm{meV}$ at $B \sim 10 \mathrm{~T}$.

We describe the hysteresis in the following way: when the bias is increased in the LCS, the $e-e(h-h)$ separation decreases until $d_{e-e} \sim d_{e-h} \approx 25 \mathrm{~nm}$; at this point, the wavefunction overlap activates screening, and the indirect excitons dissociate; in contrast, when the bias is decreased in the HCS, $d_{e-e}$ increases until the exciton gas forms. The latter transition could occur at a lower bias than the former one because screening suppresses the formation of indirect excitons. In other words, the system has a memory that arises from the interplay between screening and exciton binding.

Moreover, our measurements (Figs. 2 and 3) suggest that the transition is characterized by a strong nonlinearity of the chemical potential $\mu_{e-h}(n)(=V / e)$, which in our structure would be accompanied by a self-consistent rearrangement of the band profile. We notice that the magnetic field behavior of the width of the bistability (Fig. 4) follows strikingly the behavior of the critical temperature for the excitonic state, ${ }^{2,3}$ 
but an interpretation of our data in terms of collective properties of the indirect excitons is beyond the purposes of this paper.

Although our pictures involves mobile charges, we cannot exclude the possibility that some of the excitons are localized at interface defects, but this would not modify essentially the description. We believe that localization to defects alone (whether of excitons or free carriers) can difficultly explain the particular conditions at which the feature is observed $\left(d_{e-e} \sim d_{e-h}\right)$, the filling factor dependence of the hysteresis, and the difference in the DOS between HCS and LCS (Fig. 2).

In conclusion, we have reported on a new type of discontinuity and bistability in the tunnel current of strongly inter- acting 2D $e$ and $h$ layers obtained in single barrier GaAs/ AlAs heterostructures. These features correspond to a discontinuity in the $e-h$ density and in the phase of the current magneto-oscillations. We interpret our results in terms of a transition between the uncoupled 2DEG-2DHG and a dense gas of spatially indirect excitons.

This work is part of a research program of the "Stichting voor Fundamenteel Onderzoek der Materie', (FOM) financially supported by NWO (The Netherlands). It has also been supported by the European Commission under Contract No. ERBFMGECT950079. We are grateful to Yu. E. Lozovik, A. MacDonald, V. Volkov, M. Fromhold, and F. Sheard for useful discussions.
${ }^{1}$ Yu.E. Lozovik and O.L. Berman, Zh. Éksp. Ter. Fiz. 110, 1879 (1997) [JETP 84, 1027 (1997)]; Yu.E. Lozovik, O.L. Berman, and V.G. Tsvetus, Pisma Zh. Éksp. Ter. Fiz. 66, 332 (1997) [JETP Lett. 66, 355 (1997)].

${ }^{2}$ Y. Kuramoto and C. Horie, Solid State Commun. 25, 713 (1978).

${ }^{3}$ I.V. Lerner and Yu.E. Lozovik, J. Low Temp. Phys. 38, 333 (1980).

${ }^{4}$ X.M. Chen and J.J. Quinn, Phys. Rev. Lett. 67, 895 (1991).

${ }^{5}$ D. Yoshioka and A.H. MacDonald, J. Phys. Soc. Jpn. 59, 4211 (990).

${ }^{6}$ T. Fukuzawa, E.E. Mendez, and J.M. Hong, Phys. Rev. Lett. 64, 3066 (1990); J.A. Kash et al., ibid. 66, 2247 (1991).

${ }^{7}$ L.V. Butov, A. Zrenner, G. Abstreiter, G. Böhm, and G. Weimann, Phys. Rev. Lett. 73, 304 (1994); L.V. Butov and A.I. Filin, Phys. Rev. B 58, 1980 (1998).

${ }^{8}$ B.R.A. Neves et al., in Proceedings of the $23 \mathrm{rd}$ International Conference on Physics of Semiconductors, Berlin, 1996, edited by M. Scheffler and R. Zimmermann (World Scientific, Singapore, 1996), p. 2275.
${ }^{9}$ A. Parlangeli et al., Phys. Status Solidi A 164, 587 (1997); Physica B 256-258, 531 (1998).

${ }^{10}$ E. Böckenhoff, K.v. Klitzing, and K. Ploog, Phys. Rev. B 38, R10 120 (1988); K.S. Chan, F.W. Sheard, G.A. Toombs, and L. Eaves, ibid. 14, 47 (1997).

${ }^{11}$ R.K. Hayden et al., J. Phys. Soc. Jpn. 66, 2228 (1997).

${ }^{12}$ R. Teisser et al., Phys. Rev. B 51, 5562 (1995).

${ }^{13}$ T.M. Fromhold, F.W. Sheard, and G.A. Toombs, Surf. Sci. 228 , 437 (1990)

${ }^{14}$ M.A. Lampert and P. Mark, Current Injection in Solids (Academic Press, New York, 1970).

${ }^{15}$ J.J. Finley et al., Superlattices Microstruct. 23, 513 (1998).

${ }^{16}$ O.V. Volkov, V.E. Zhitomirskii, I.V. Kukushkin, K. von Klitzing, and K. Eberl, Pisma Zh. Éksp. Teor. Fiz. 64, 719 (1996) [JETP Lett. 64, 774 (1996)].

${ }^{17}$ A comprehensive study of a gas of indirect excitons in highmagnetic fields has been recently reported by Yu.E. Lozovik and A.M. Ruvinsky, Phys. Lett. A 227, 271 (1997).

${ }^{18}$ S.M. Cao and M. Willander, J. Appl. Phys. 81, 6221 (1997). 